\title{
Avaliação dos efeitos do estradiol e do FSH nos niveis de leptina em mulheres com supressão da função hipofisária
}

\author{
Effects of estradiol and FSH on leptin levels in women with pituitary suppression
}

Selmo Geber ${ }^{1}$, Marcos Sampaio ${ }^{2}$

\section{RESUM0}

Objetivo: identificar a correlação entre os níveis séricos de leptina e os níveis de estradiol e do hormônio folículo-estimulante (FSH) em mulheres com supressão da função hipofisária, e suas possíveis interferências no eixo reprodutivo. Métodos: estudamos prospectivamente 64 pacientes submetidas à hiperestimulação ovariana controlada com FSH recombinante para tratamento pela técnica de reprodução assistida, devido a fator masculino ou tubário, e 20 pacientes em uso de valerato de estradiol, para preparo endometrial, em tratamento de doação de óvulos, por falha de resposta ovariana em ciclo prévio. Todas as pacientes utilizaram análogo de GnRH no início do tratamento, de forma a obter a supressão da função hipofisária. Para a análise estatística dos resultados, foram utilizados os testes $\chi^{2}, t$ de Student e correlação de Pearson, quando adequado. Os resultados foram considerados significativos quando $p<0,05$. Resultados: observamos que os níveis de leptina correlacionaram-se com o índice de massa corpórea, embora não tenham influenciado a taxa de crescimento desses hormônios. Identificamos correlação positiva entre os níveis médios de estradiol e leptina em ambos os grupos, com os níveis desta passando de 10,42 ng/mL para $15,68 \mathrm{ng} / \mathrm{mL}$ no grupo $\mathrm{FSH}$ e de $11,09 \mathrm{ng} / \mathrm{mL}$ para $14,5 \mathrm{ng} / \mathrm{mL}$ no grupo estradiol, com a elevação dos níveis de estradiol. As taxas de crescimento da leptina foram mais altas nas mulheres que apresentaram níveis mais elevados de estradiol sérico, isto é, que tiveram ciclos induzidos com FSH recombinante, do que nas que usaram valerato de estradiol $(159,60 \pm 58,01$ e 136,73 $\pm 34,17$, respectivamente). Conclusões: podemos concluir que o estradiol é importante regulador da produção da leptina, podendo ter seu efeito amplificado por seus elevados níveis séricos ou pela associação com o FSH.

PALAVRAS-CHAVE: Leptina; Estradiol; Hormônio folículo estimulante; Infertilidade

\section{ABSTRACT}

Purpose: to identify the relationship between serum levels of leptin and the levels of estradiol and follicle-stimulating hormone (FSH) in women with pituitary suppression and to evaluate its possible interference on the reproductive axis. Methods: a total of 64 patients submitted to controlled ovarian hyperstimulation with recombinant FSH for assisted reproduction, due to a male or tubal factor, and 20 patients using estradiol valerate, for endometrial preparation in order to be submitted to oocyte donation treatment were studied. All patients used GnRH analogues before starting treatment in order to avoid premature LH surge. Data were analyzed statistically by the $\chi^{2}$ test, Student's $t$-test and the Pearson correlation test, when appropriate, with the level of significance set at $p<0,05$. Results: it was observed that leptin levels correlated with body mass index (BMI) even though they had not influenced growth rate of these hormones. A positive correlation was observed between estradiol and leptin levels in both groups, as leptin levels increased from 10.42 to 15.68 $\mathrm{ng} / \mathrm{mL}$ in the FSH group and from 11.09 to $14.5 \mathrm{ng} / \mathrm{mL}$ in the estradiol group, following estradiol increase. The growth rate of leptin was higher in women with higher estradiol levels, i.e., who had induced cycles with recombinant FSH, than in those who received estradiol valerate ( $159.60 \pm 58.01$ and $136.73 \pm 34.17$, respectively). Conclusion: we may state that leptin correlated with BMI and that both FSH and estradiol do interfere in the regulation of leptin production in women.

KEYWORDS: Leptin; Estradiol; Follicle stimulating hormone; Infertility

Clínica ORIGEN - Centro de Medicina Reprodutiva - Belo Horizonte (MG).

1,2 Diretor da Clínica ORIGEN - Centro de Medicina Reprodutiva - Belo Horizonte (MG).

Correspondência: Selmo Geber

Av. Contorno, 7747 - Lourdes - 30110-120 - Belo Horizonte - MG - e-mail: selmogeber@origen.com.br

Recebido em: 22/11/2004 Aceito com modificações em: 7/4/2005

Rev Bras Ginecol Obstet. 2005; 27(4):216-21 


\section{Introdução}

A correlação entre a gordura corporal e os fenômenos envolvidos nos processos da reprodução vem instigando pesquisadores desde os tempos mais remotos. A fertilidade depende de uma nutrição adequada e das reservas de energia, havendo, portanto, intima relação entre as reservas metabólicas e a capacidade reprodutiva. Como exemplo deste fenômeno, observa-se que tanto as pessoas em restrição dietética extrema quanto as excessivamente obesas apresentam alterações no eixo reprodutivo. Além disso, mulheres obesas desenvolvem a menarca mais precocemente em relação às mulheres de peso normal ${ }^{1}$. Uma teoria para explicar esse fenômeno é a de que a menstruação seja iniciada quando o peso atinge uma massa crítica, a qual as mulheres obesas alcancem primeiro e que seria de $48 \mathrm{~kg}$ ou aproximadamente $22 \%$ de gordura corporal ${ }^{2}$.

Mulheres muito magras, com bulimia ou anorexia nervosa e atletas ou bailarinas submetidas a exercícios extenuantes apresentam irregularidades no ciclo menstrual ${ }^{3}$. Por outro lado, mulheres muito obesas também podem apresentar alterações na sua função reprodutiva, como observado na síndrome dos ovários policísticos ${ }^{4}$, freqüentemente associada à infertilidade, obesidade e hiperandrogenismo.

A leptina é proteína codificada pelo gene da obesidade (ob), secretada pelos adipócitos e recentemente descoberta por Zhang et al. ${ }^{5}$. Atua no sistema nervoso central como fator de sinalização para regular a homeostase do peso corporal e o estado de reserva energética do organismo ${ }^{6-8}$. Primeiramente, pensava-se que fosse um hormônio antiobesidade, mas existem evidências de que participa de vários processos metabólicos e endócrinos, incluindo a função reprodutiva ${ }^{9}$.

A presença de receptores para leptina em todos os niveis do eixo hipotálamo-hipófise-ovariano sugere uma correlação entre os mecanismos de regulação envolvendo os processos de nutrição e reprodução, talvez em uma rede complexa de interações em múltiplos níveis, de maneira parácrina e/ou endócrina.

Embora receptores de leptina tenham sido demonstrados em ovários humanos, não há informações sobre os efeitos da mesma nas células foliculares ${ }^{2,10}$. É possivel que o estradiol e o hormônio folículo-estimulante (FSH) interfiram na regulação da produção de leptina pelos adipócitos ${ }^{11}$. No entanto, os resultados ainda são conflitantes ${ }^{9,12,13}$.

O objetivo de nosso estudo foi identificar as correlações e influências do estrogênio e do FSH sobre os niveis circulantes de leptina, em mulhe- res com supressão da função hipofisária e conseqüentemente sem efeito e interferência das outras gonadotrofinas e esteróides ovarianos.

\section{Métodos}

Foram incluídas prospectivamente 84 pacientes com o diagnóstico de infertilidade. Dessas, 64 tinham como indicação para o tratamento o fator tubário ou masculino (isoladamente) e 20 tinham indicação para tratamento com doação de óvulos. Após esclarecimentos sobre a finalidade do trabalho, as pacientes incluídas no estudo assinaram o consentimento. O estudo foi aprovado pelo Comitê de Ética Institucional, estando de acordo com as normas éticas e legais vigentes no país.

O diagnóstico de infertilidade tubária foi dado após confirmação de obstrução tubária bilateral identificada à histerossalpingografia ou à videolaparoscopia, associada ao teste de cromotubagem com azul de metileno, independentemente de sua localização. O diagnóstico do fator masculino foi dado após a análise de dois espermogramas realizados com intervalo de 60 dias, baseado nos critérios da OMS ${ }^{14}$. O diagnóstico de baixa reserva ovariana foi dado quando os niveis de $\mathrm{FSH}$ encontravam-se superiores a $15 \mathrm{mUI} / \mathrm{mL}$ e os de estradiol inferiores a $60 \mathrm{pg} / \mathrm{mL}$, medidos no terceiro dia do ciclo menstrual. Nestes casos realizou-se tratamento com doação de óvulos.

O índice de massa corporal (IMC) foi calculado pelo quociente do peso (em kg) pela estatura (em metros), ao quadrado. Para fins de comparação, foram consideradas obesas as pacientes com IMC superior a 25.

As pacientes foram divididas em dois grupos: grupo FSH: neste grupo foram incluidas as pacientes $(n=64)$ com reserva folicular adequada ( $\mathrm{FSH}<15$ $\mathrm{mUI} / \mathrm{mL}$ ), sem alterações hormonais, sendo a indicação para tratamento de fertilização in vitro o fator masculino ou tubário; todas foram submetidas ao mesmo protocolo de indução da superovulação; grupo Estradiol: neste grupo foram incluídas as pacientes $(n=20)$ submetidas a tratamento de doação de oócitos. Todas foram submetidas ao mesmo protocolo para preparo endometrial.

As amostras de sangue para dosagem de estradiol e da leptina foram colhidas entre 8 e 10 horas da manhã, no dia em que havia comprovação da supressão hipofisária (pré-tratamento) (grupos FSH e Estradiol) e no dia da administração do hCG (grupo FSH) ou no dia da inseminação in vitro, antes do início do uso do progesterona (grupo Estradiol), que correspondem aos momentos relativos aos niveis mais altos de estradiol (pós-tratamento). 
Os níveis séricos de FSH e estradiol foram obtidos por radioimunoensaio (Amerlite FSH e Amerlite Estradiol-60). Os resultados foram expressos como unidades internacionais por litro (UI/L). A leptina foi avaliada em todas as amostras utilizando-se também o radioimunoensaio como método de análise. Os kits continham anticorpo contra leptina humana preparado em coelhos (Linco Research, St. Charles, MO, USA). Os resultados foram em nanogramas por mililitro $(\mathrm{ng} / \mathrm{mL})$.

As pacientes do grupo FSH que se submeteram à indução da superovulação iniciaram o tratamento com a administração de análogo do hormônio liberador das gonadotrofinas (GnRHa Zoladex, Astrazeneca, Brasil) por via subcutânea, no $2^{\circ}$ ou no $21^{\circ}$ dia do ciclo menstrual, para obtenção da supressão hipofisária ${ }^{15}$. Aproximadamente 10 dias após, realizaram-se dosagem de estradiol sérico e ultra-sonografia endovaginal para confirmação do bloqueio hipofisário. Quando os níveis de estradiol estavam menores que $30 \mathrm{pg} / \mathrm{mL}$ e à ultra-sonografia identificava-se o endométrio com espessura menor do que $5 \mathrm{~mm}$ e ausência de cistos ovarianos, a supressão hipofisária era confirmada e a paciente era considerada pronta para o início da indução da superovulação. Para a indução foi utilizado o FSH recombinante (Puregon, Organon, Brasil), e a dose inicial variou de acordo com a idade da paciente, variando de 200 a 300 UI ao dia, por via subcutânea, durante quatro dias. A partir daí, as doses foram individualizadas e adequadas de acordo com a resposta de cada paciente. A monitorização da resposta ovariana foi feita pela dosagem dos níveis séricos de estradiol e pela ultra-sonografia endovaginal para acompanhamento do desenvolvimento folicular e espessamento endometrial. Quando pelo menos dois folículos apresentavam-se com diâmetro médio de $17 \mathrm{~mm}$ associados a niveis compativeis de estradiol, a maturação oocitária era induzida por meio da administração de 10.000 UI do hormônio da gonadotrofina coriônica (hCG - Profasi, Serono, Brasil) por via intramuscular. Aproximadamente 34 horas após, as pacientes foram submetidas à punção folicular guiada por ultra-som, para captação oocitária.

As pacientes do grupo Estradiol submetidas a tratamento com doação de oócitos iniciaram o preparo endometrial com administração de GnRHa por via subcutânea, no $2^{\circ}$ ou $21^{\circ}$ dia do ciclo menstrual, para a obtenção da supressão hipofisária. Aproximadamente 10 dias após, realizaram dosagem de estradiol sérico e ultra-sonografia endovaginal para confirmação do bloqueio hipofisário. Quando os níveis de estradiol estavam mais baixos que $30 \mathrm{pg} /$ $\mathrm{mL}$ e à ultra-sonografia identificava-se o endométrio com espessura menor do que $5 \mathrm{~mm}$ e ausência de cistos ovarianos, a supressão hipofisária era confirmada e a paciente era considerada pronta para o início do preparo endometrial. O preparo endometrial era feito utilizando-se valerato de estradiol (Climene, Schering, Brasil), com $2 \mathrm{mg}$ diariamente, aumentando-se a dose em $2 \mathrm{mg}$ a cada cinco dias até alcançar a dose de $6 \mathrm{mg}$ ao dia ${ }^{16}$. Após 15 dias de uso, realizavam-se nova dosagem de estradiol sérico e nova ultra-sonografia para confirmar o adequado preparo endometrial para a transferência embrionária. Quando os níveis de estradiol estavam maiores que $250 \mathrm{pg} / \mathrm{mL}$ e a espessura endometrial estava maior que $8 \mathrm{~mm}$, as pacientes eram consideradas prontas para a transferência embrionária.

Todos os exames foram realizados pelo mesmo examinador, no período de 8 às 10 horas da manhã, utilizando-se o mesmo equipamento de ultrasom bidimensional, computadorizado, com transdutor eletrônico, multifreqüencial, na freqüência de 6,5 $\mathrm{MHz}$ (Tosbee, Toshiba, Japão). Para a confirmação do bloqueio hiposfisário, o exame foi realizado 10 dias após o uso de GnRHa em ambos os grupos, juntamente com a dosagem de estradiol. Nas pacientes submetidas à indução da superovulação, a ultrasonografia foi repetida a cada quatro dias para controle do desenvolvimento folicular. No grupo Estradiol a ultra-sonografia foi repetida 15 dias após o início do uso do valerato de estradiol.

Para a análise estatística dos resultados, foi utilizado o teste $\chi^{2}$ para comparação entre os dois grupos. A comparação entre o IMC e os grupos com relação às médias dos valores de estradiol, da leptina e de sua taxa de elevação (leptina inicial/ leptina final x 100) foi feita pelo teste $t$ de Student, que também foi usado para verificar se houve aumento nos niveis de estradiol e leptina durante o período de estudo. Além disso, utilizamos a correlação de Pearson, como forma de avaliar a relação entre duas variáveis de interesse. Todos os resultados foram considerados significativos quando $\mathrm{p}<0,05$.

\section{Resultados}

As 84 pacientes que iniciaram o estudo completaram o tratamento e nenhuma apresentou efeitos adversos. A idade das pacientes do grupo FSH variou de 27 a 42 anos, com média de $34,3 \pm 3,71$ anos, e no grupo Estradiol, de 31 a 50 anos, com média de $42 \pm 4,8$ anos. Quando comparados os dois grupos, o resultado obtido demonstrou que a idade das pacientes do grupo Estradiol foi significativamente superior $(\mathrm{p}<0,001)$ ao encontrado nas pacientes do grupo FSH. Todas as pacientes apresentaram niveis de hormônio tirotrófico e prolactina adequados para a idade e fase do ciclo. 
Para a avaliação dos efeitos da obesidade, calculamos o IMC e os resultados obtidos demonstraram que no grupo FSH havia 11 pacientes obesas $(17,2 \%)$ e no grupo Estradiol, 3 obesas (15\%). A comparação entre os grupos mostrou semelhança na incidência de mulheres obesas (Tabela 1). Quando avaliamos a variação dos níveis de leptina sérica de acordo com o IMC, observamos que as obesas apresentaram níveis séricos de leptina pré e pós-tratamento significativamente mais altos do que as não obesas.

Tabela 1 - Comparação entre as idades e os índices antropométricos das pacientes estudadas.

\begin{tabular}{lccl}
\hline Variável & Grupo FSH & Grupo Estradiol & $\mathbf{p}$ \\
\hline Idade (anos) & $34,30 \pm 3,71$ & $42,00 \pm 4,80$ & $<0,001$ \\
Peso $(\mathrm{kg})$ & $62,20 \pm 11,65$ & $59,60 \pm 13,37$ & 0,40 \\
Altura $(\mathrm{m})$ & $1,63 \pm 0,07$ & $1,61 \pm 0,05$ & 0,24 \\
IMC & $23,04 \pm 5,10$ & $23,28 \pm 4,08$ & 0,85 \\
\hline
\end{tabular}

Valores são média \pm desvio padrão.

Teste $t$ de Student.

IMC = índice de massa corpórea.

A análise dos níveis de estradiol sérico realizada antes do início do tratamento, isto é, na fase de supressão da função hipofisária (pré-tratamento), comparada aos niveis obtidos no dia da administração do hCG (pós-tratamento), mostrou aumento significativo $(\mathrm{p}=0,0001)$ para ambos os grupos de estudo (Tabela 2). No grupo FSH os níveis de estradiol passaram de 16,75 $\pm 7,81$ (variação de 9,00 a 29,00 ) para 1.440,36 $1.122,55$ (variação de 200,00 a 5.150,00). No grupo Estradiol, os níveis

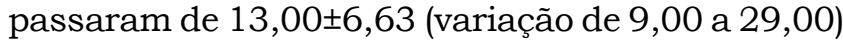

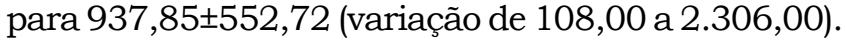

Tabela 2 - Comparação entre os níveis de estradiol sérico, pré e pós-tratamento.

\begin{tabular}{lccc}
\hline & Estradiol pré & Estradiol pós & $\mathbf{p}$ \\
\hline Grupo FSH & $16,75 \pm 7,81$ & $1.440,36 \pm 1.122,55$ & 0,0001 \\
& $(9,00-29,00)$ & $(200,00-5.150,00)$ & 0,0001 \\
Grupo Estradiol & $13,00 \pm 6,63$ & $937,85 \pm 552,72$ & \\
& $(9,00-29,00)$ & $(108,00-2.306,00)$ & \\
\hline
\end{tabular}

Valores são média \pm desvio padrão (variação).

Teste $t$ de Student para amostras pareadas.

Quando comparamos os niveis de leptina sérica, medidos nos mesmos dias da dosagem de estradiol, isto é, período pré e pós-tratamento, observamos um aumento significativo nos niveis dosados pós-tratamento ( $\mathrm{p}=0,0001$ e 0,0079$)$ para ambos os grupos de estudo, respectivamente (Tabela 3). No grupo FSH os níveis de estradiol passaram de 10,42 $\pm 6,81$ (variação de 2,10 a 45,00) para $15,68 \pm 9,56$ (variação de 2004,60 a 46,20). No gru- po Estradiol, os niveis passaram de $11,09 \pm 9,99$ (variação de 2,30 a 40,40 ) para $14,45 \pm 12,48$ (variação de 3,00 a 53,80$)$.

Tabela 3 - Comparação entre os níveis de leptina sérica, pré e pós-tratamento.

\begin{tabular}{lllc}
\hline & Leptina pré & Leptina pós & p \\
\hline Grupo FSH & $10,42 \pm 6,81$ & $15,68 \pm 9,56$ & 0,0001 \\
& $(2,10-45,00)$ & $(4,60-46,20)$ & \\
Grupo Estradiol & $11,09 \pm 9,99$ & $14,45 \pm 12,48$ & 0,0079 \\
& $(2,30-40,40)$ & $(3,00-53,80)$ & \\
\hline
\end{tabular}

Valores são média \pm desvio padrão (variação).

Teste $t$ de Student para amostras pareadas.

Com o objetivo de avaliar se os hormônios estudados apresentavam alguma correlação entre si, utilizamos a análise de correlação de Pearson. Os níveis de leptina sérica pré e pós-tratamento apresentaram coeficiente de correlação de 0,72 para o grupo FSH e de 0,92 para o grupo Estradiol, confirmando a significância estatística para ambos os grupos $(p=0,0001)$. Esse resultado demonstra que os níveis finais de leptina estão diretamente relacionados com os niveis presentes inicialmente.

Para avaliar a taxa de elevação dos níveis de leptina sérica do primeiro para o segundo exame, dividimos o valor encontrado na dosagem pós-tratamento pelo valor encontrado na dosagem pré-tratamento, sendo o resultado multiplicado por 100 . Quando comparamos as taxas de elevação da leptina nos grupos de mulheres obesas e não obesas, não observamos diferença significativa $(\mathrm{p}=0,7510)$.

Por fim, comparamos os resultados obtidos nas pacientes do grupo FSH com os das pacientes do grupo Estradiol, e não identificamos diferenças entre os níveis séricos de leptina pré e pós-tratamento (Tabela 4). Entretanto, quando avaliamos os niveis de estradiol pré e pós-tratamento e a taxa de elevação da leptina, observamos niveis significativamente maiores nas pacientes do grupo FSH.

Tabela 4 - Comparação entre as pacientes dos grupos FSH e Estradiol em relação aos niveis de estradiol e leptina sérica, pré e pós-tratamento.

\begin{tabular}{lccc}
\hline & Grupo FSH & Grupo Estradiol & $\mathbf{p}$ \\
\hline Estradiol pós & $1.440,36 \pm 1.122,55$ & $937,85 \pm 552,72$ & 0,0091 \\
& $(200,00-5.150,00)$ & $(108,00-2.306,00)$ & \\
Leptina pré & $10,42 \pm 6,81$ & $11,09 \pm 9,99$ & 0,7827 \\
& $(2,10-45,00)$ & $(2,30-40,40)$ & \\
Leptina pós & $15,68 \pm 9,56$ & $14,45 \pm 12,48$ & 0,6907 \\
& $(4,60-46,20)$ & $(3,00-53,80)$ & \\
Taxa de crescimento & $159,60 \pm 58,01$ & $136,73 \pm 34,17$ & 0,0342 \\
da leptina & $(74,22-408,85)$ & $(65,36-213,75)$ & \\
\hline
\end{tabular}

Valores são média \pm desvio padrão (variação).

Teste $t$ de Student para amostras independentes.

Taxa de crescimento da leptina $=($ leptina-pós/leptina-pré $) \times 100$. 


\section{Discussão}

A leptina é polipeptídeo que está implicado na regulação de vários processos fisiológicos, atuando na promoção da maturação sexual, exercendo influência no eixo hipotalâmico-hipofisáriogonadal $^{8}$. Sabe-se que a sintese e liberação da leptina é feita pelos adipócitos, estando relacionada ao IMC, atuando diretamente no centro da saciedade e controlando assim a ingestão calórica. Como existe correlação entre a maturação sexual e o IMC, aventou-se a possibilidade de uma interação entre a leptina e os hormônios do eixo hiptálamo-hipófise-ovariano. De fato, alguns estudos tentaram demonstrar uma relação entre estradiol, FSH e a produção de leptina pelos adipócitos ${ }^{11}$, sem contudo apresentar resultados definitivos ${ }^{9,12,13}$. Nosso estudo analisou, talvez pela primeira vez, a interação da leptina com o estradiol e o FSH, sem que houvesse interferência dos demais hormônios hipofisários e ovarianos. Essa situação ideal foi obtida devido ao bloqueio da função hipofisária após a administração dos GnRHa.

$\mathrm{O}$ fato de que as mulheres do grupo Estradiol tinham media etária superior era esperado, uma vez que as pacientes deste grupo estavam em programa de doação de óvulos devido à história de baixa ou ausência de resposta ovariana à indução, fato associado ao aumento da idade.

Em relação à obesidade, os dois grupos apresentaram a mesma proporção de obesas, não criando, assim, viés para comparação entre os grupos. Quando avaliamos a variação dos niveis de leptina sérica de acordo com o IMC, os resultados mostraram que as obesas apresentaram niveis séricos de leptina pré e pós-tratamento significativamente mais altos do que as não obesas, resultados estes em acordo com o observado na literatura ${ }^{17}$.

A comparação feita entre os niveis séricos de estradiol pré e pós-tratamento mostrou aumento significativo no período pós-tratamento em ambos os grupos, confirmando o que já se esperava, pois as pacientes tiveram sua foliculogênese induzida ou receberam o estradiol diretamente, e no período prétratamento apresentavam-se com hipoestrogenismo induzido pelo uso do análogo do GnRH. Quando comparamos os níveis de estradiol sérico no período póstratamento, dos dois grupos, observamos niveis significativamente superiores no grupo FSH. Este fato ocorreu uma vez que as mulheres deste grupo foram submetidas a estimulo ovariano e os niveis observados eram decorrentes de sua resposta ovariana. No grupo Estradiol, as pacientes receberam doses fixas de valerato de estradiol, assim, os níveis séricos identificados tinham um limite correspondente à dose administrada.

Com relação aos níveis de leptina, também observamos aumento significativo no pós-tratamen- to em relação ao pré-tratamento, em ambos os grupos. Esses achados confirmaram, pela primeira vez, elevação nos níveis de leptina secundária ao aumento do estradiol em pacientes não expostas aos demais hormônios ovarianos e hipofisários (GnRH dependentes), sugerindo correlação direta entre a elevação do estradiol e da leptina, e que o estradiol possa ter um papel estimulador da produção ou liberação de leptina. Um provável mecanismo de ação para explicar este fenômeno é o incremento da expressividade do gene ob (regulador da síntese da leptina pelos adipócitos), induzida pelo estradiol.

Resultados semelhantes já foram obtidos em outros estudos realizados com mulheres submetidas à hiperestimulação ovariana para fertilização in vitro sem uso de GnRHa ${ }^{18-21}$. A grande diferença entre os estudos está no fato de que as pacientes estavam sob a ação de todos os hormônios ovarianos e hipofisários, o que poderia levar a possivel interferência ou viés nos resultados. Entendemos que os resultados aqui apresentados são mais consistentes, uma vez que todas as pacientes tiveram a função da hipófise suprimida previamente, não havendo, assim, interferência dos outros hormônios hipofisários e ovarianos.

Os niveis séricos de leptina foram semelhantes em ambos os grupos tanto no período pré quanto no pós-tratamento, o que novamente demonstra a semelhança entre os dois grupos. Em relação às taxas de elevação da leptina, entretanto, identificamos aumento significativo no padrão de elevação dos niveis hormonais das pacientes que tiveram seus ciclos induzidos por FSHr, (grupo FSH) quando comparadas com o grupo Estradiol. Este resultado pode ser explicado pelos maiores níveis de estradiol no pós-tratamento, também observados neste grupo de pacientes. Entretanto, não podemos também descartar a possibilidade de uma ação direta do FSH (presente apenas no grupo FSH) sobre a produção de leptina, por possivel produção ovariana ou interferência indireta na produção deste hormônio pelos adipócitos, por meio de outras substâncias ovarianas ${ }^{22,23}$.

Esta afirmação, entretanto, ainda pode ser considerada controversa, uma vez que Rosenbaum et al. ${ }^{24}$ e Shimizu et al. ${ }^{21}$ demonstraram que mulheres na menopausa apresentam niveis de leptina sérica mais baixos do que durante a menacme (grupo controle). Como na menopausa os níveis endógenos de FSH estão bastante elevados, esses dados podem questionar a ação desse hormônio sobre a leptina.

Por outro lado, outros autores ${ }^{2,22}$ demonstraram a presença de receptores de leptina em folículos ovarianos e a expressão do RNAm nas células da granulosa e cumulus oophorus. Assim, é possivel que a influência do FSH sobre a produção ovariana de leptina ocorra somente na presença de folículos, fato que não ocorre na menopausa. 
Concluindo, o presente estudo demonstra provavelmente pela primeira vez que o estradiol é importante regulador da produção da leptina, podendo ter seu efeito amplificado por seus elevados níveis séricos ou pela associação com o FSH.

Esses resultados podem contribuir para o maior conhecimento da interação entre os processos metabólicos e os fenômenos envolvidos no processo de reprodução.

\section{Referências}

1. Bray GA. Obesity and reproduction. Hum Reprod. 1997;12 Suppl 1:26-32.

2. Karlsson C, Lindell K, Svensson E, Bergh C, Lind $\mathrm{P}$, Billig $\mathrm{H}$, et al. Expression of functional leptin receptors in the human ovary. J Clin Endocrinol Metab. 1997;82(12):4144-8.

3. Abraham SF, Beumont PJ, Fraser IS, Llewellyn-Jones D. Body weight, exercise and menstrual status among ballet dancers in training. Br J Obstet Gynaecol. 1982;89(7):507-10.

4. Melo MAB, Sabino SM, Sampaio MAC, Geber S. Leptin effects on polycystic ovarian syndrome. Hum Reprod. 2002;17 Suppl 1:45-6.

5. Zhang Y, Proença R, Maffei M, Barone M, Leopold L, Friedman JM. Positional cloning of the mouse obese gene and its human homologue. Nature. 1994;372(6505):425-32.

6. Campfield LA, Smith FJ, Guisez Y, Devos R, Burn P. Recombinant mouse OB protein: evidence for a peripheral signal linking adiposity and central neural networks. Science. 1995;269(5223):546-9.

7. Pelleymounter MA, Cullen MJ, Baker MB, Hecht R, Winters D, Boone T, et al. Effects of the obese gene product on body weight regulation in ob/ob mice. Science. 1995;269(5223):540-3.

8. Clarke IJ, Henry BA. Leptin and reproduction. Rev Reprod. 1999;4(1):48-55.

9. Messinis IE, Milingos SD, Alexandris E, Kariotis I, Kollios G, Seferiadis K. Leptin concentrations in normal women following bilateral ovariectomy. Hum Reprod. 1999;14(4):913-8.

10. Spicer LJ, Francisco CC. The adipose obese gene product, leptin: evidence of a direct inhibitory role in ovarian function. Endocrinology. 1997;138(8):3374-9.

11. Bouvattier C, Lahlou N, Roger M, Bougneres P. Hyperleptinaemia is associated with impaired gonadotrophin responses to $\mathrm{GnRH}$ during late puberty in obese girls, not boys. Eur J Endocrinol. 1998;138(6):653-8.

12. Rechberger T, Baranowski W, Postawski K, Jakimiuk AJ, Tomaszewski J, Kulik-Rechberger B, et al. Serum leptin concentrations in women taking oral contraceptives. Eur J Obstet Gynecol Reprod Biol. 1999;83(1):105-8.

13. Riad-Gabriel MG, Jinagouda SD, Sharma A, Boyadjian R, Saad MF. Changes in plasma leptin during the menstrual cycle. Eur J Endocrinol. 1998;139(5):528-31.

14. World Health Organization. WHO laboratory manual for the examination of human semen and sperm-cervical mucus interaction. 3rd ed. Cambridge: Cambridge University Press; 1993.

15. Geber S, Sales L, Sampaio MA. Comparison between a single dose of goserelin (depot) and multiple daily doses of leuprolide acetate for pituitary suppression in IVF treatment: a clinical endocrinological study of the ovarian response. J Assist Reprod Genet. 2000;19(7):313-8.

16. Sampaio MA, Geber S. Births after transfer of zona-free blastocysts in oocyte donation cycles. J Assist Reprod Genet. 2001;18(3):156-9.

17. Kolaczynski JW, Nyce MR, Considine RV, Boden G, Nolan JJ, Henry R, et al. Acute and chronic effect of insulin on leptin reproduction in humans: studies in vivo and in vitro. Diabetes. 1996;45(5):699-701.

18. Messinis IE, Milingos S, Zikopoulos K, Kollios G, Seferiadis K, Lolis D. Leptin concentrations in the follicular phase of spontaneous cycles and cycles superovulated with follicle stimulating hormone. Hum Reprod. 1998;13(5):1152-6.

19. Strowitzki T, Kellerer M, Capp E, Haring HU. Increase in serum leptin concentrations in women undergoing controlled ovarian hyperstimulation for assisted reproduction. Gynecol Endocrinol. 1998;12(3):167-9.

20.Zhao Y, Kreger DO, Brannian JD. Serum leptin concentrations in women during gonadotropin stimulation cycles. J Reprod Med. 2000;45(2):121-5.

21. Shimizu H, Shimomura Y, Nakanishi Y, Futawatari $\mathrm{T}$, Ohtani K, Sato N, et al. Estrogen increases in vivo leptin production in rats and human subjects. J Endocrinol. 1997;154(2):285-92.

22. Cioffi JA, Van Blerkom J, Antczak M, Shafer A, Wittmer S, Snodgrass HR. The expression of leptin and its receptors in pre-ovulatory human follicles. Mol Hum Reprod. 1997;3(6):467-72.

23.Zachow RJ, Magoffin DA. Direct intraovarian effects of leptin: impairment of the synergistic action of insulin-like growth factor-I on follicle-stimulating hormone-dependent estradiol-17 beta production by rat ovarian granulosa cells. Endocrinology. 1997;138(2):847-50.

24. Rosenbaum M, Nicolson M, Hirsch J, Heymsfield SB, Gallagher D, Chu F, et al. Effects of gender, body composition, and menopause on plasma concentrations of leptin. J Clin Endocrinol Metab. 1996;81(9):3424-7. 